\title{
NONLINEAR OSCILLATIONS IN NEW ANHARMONIC POTENTIAL
}

\author{
H. Konwent, P. Machnikowski and A. Radosz \\ Institute of Physics, Technical University of Wrocław \\ Wybrzeże Wyspiańskiego 27, 50-370 Wrocław, Poland
}

(Received November 21, 1995; in final form February 8, 1996)

The classical equation of motion for a particle moving in the new double-well potential $V(x)=\frac{1}{2} V_{0}(A \cosh a x-1)^{2}$ is solved exactly for different values of the parameter $A$ and the energy constant $E$. The solutions in various special cases are discussed.

PACS numbers: 03.20.+i

\section{Introduction}

Strongly anharmonic potentials are of great interest in various branches of physics. The best known one-dimensional potential is the potential of the form

$$
U(x)=-\frac{1}{2} A x^{2}+\frac{1}{4} B x^{4}+\frac{A^{2}}{4 B},
$$

which for $A>0$ and $B>0$ is a double-well potential. It is well known (see e.g. [1]) that the classical equation of motion

$$
\frac{\mathrm{d}^{2} x}{\mathrm{~d} t^{2}}=-\frac{\mathrm{d} V(x)}{\mathrm{d} x}
$$

for the potential (1) can be integrated with the use of the energy integral

$$
E=\frac{m}{2}\left(\frac{\mathrm{d} x}{\mathrm{~d} t}\right)^{2}+V(x) .
$$

Recently the anharmonic potential of the form

$$
V(x)=\frac{1}{2} V_{0}(A \cosh a x-1)^{2},
$$

where $V_{0}, A$ and $a$ are the positive constants, has been studied [2]. This potential is related to the double Morse potential, used in the theory of hydrogen bonded ferroelectrics [3], and to the Razavy potential [4]. Similar potential has been proposed by Zaslavsky and Ulyanov [5]. 
This potential has an interesting property. For some values of the parameter $V_{0}$ the one-dimensional Schrödinger equation can be solved exactly and a few energy levels and the corresponding wave functions can be found in analytical form. Hence, the potential (4) belongs to the class of quasi exactly solvable potentials (investigated e.g. in Refs. [6] and [7]). However, the hidden symmetry [8] underlying this property does not appear in the classical case. It is well known that the $\phi^{4}$ potential (1) does not possess this property.

It is evident that the potential (4) is a double-well potential for $0<A<1$ (Fig. 1a) and it is a one-well strongly anharmonic potential for $A>1$ (Fig. 1c). We note that for $A=1$ the potential (4) is very flat in the vicinity of the point $x=0$ (Fig. 1b).
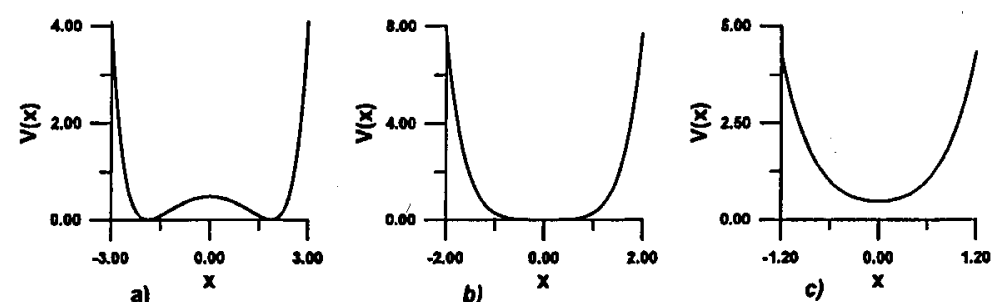

Fig. 1. The potential (4): Double-well potential, $0<A<1$ (a); Flat bottom strongly anharmonic potential, $A=1$ (b); Single-well potential, $A>1$ (c).

Introducing the notation

$$
u=a x, \quad \omega_{0}=\sqrt{\frac{V_{0} a^{2}}{m}}, \quad \varepsilon=\sqrt{\frac{2 E}{V_{0}}}, \quad \tau=\omega_{0} t,
$$

we write Eqs. (2) and (3) with the potential (4) in the form

$$
\begin{aligned}
& \frac{\mathrm{d}^{2} u}{\mathrm{~d} \tau^{2}}=-A \sinh u(A \cosh u-1), \\
& \left(\frac{\mathrm{d} u}{\mathrm{~d} \tau}\right)^{2}=\varepsilon^{2}-(A \cosh u-1)^{2} .
\end{aligned}
$$

In this paper an extensive discussion of solutions of the classical equation of motion (7) will be presented. The paper is organized as follows: in the subsequent Secs. 2-4 the equation of motion will be solved for three different forms of potential (4), whereas in Sec. 5 the case of the reversed potential is described; the final discussion and comments are given in Secs. 6 and 7.

\section{Double-well potential}

First, let us consider the case when $0<A<1$. From the equation of motion (6) written in the form

$$
\frac{\mathrm{d} u}{\mathrm{~d} \tau}=\dot{u}, \quad \ddot{u} \equiv \frac{\mathrm{d} \dot{u}}{\mathrm{~d} \tau}=-\dot{A} \sinh u(A \cosh u-1),
$$

we see that the system has the following critical points: 
- the saddle point: $u=0, \dot{u}=0$,

- two centres: $u=u_{0}, \dot{u}=0$, and $u=-u_{0}, \dot{u}=0$, where $\cosh u_{0}=\frac{1}{A}>1$.

Schematically the phase portrait is presented in Fig. 2a. The phase trajectories are closed curves, corresponding to oscillating motion. The separatrix is associated with the energy $\varepsilon=1-A$, and its two branches are described by the equation

$$
\dot{u}^{2}=(1-A)^{2}-(A \cosh u-1)^{2} .
$$

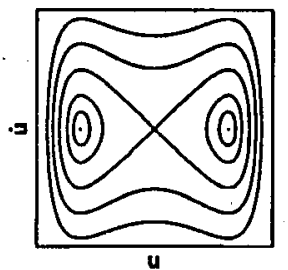

a)

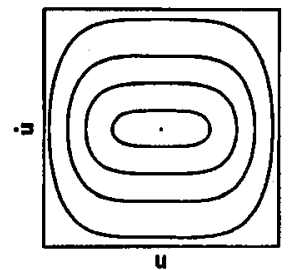

b)

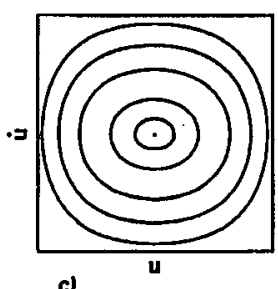

c)

Fig. 2. The phase portraits of the system for different characters of the potential: Double-well potential, $A=0.3$ (a); Flat bottom potential (b); Single-well potential, $A=1.7$ (c).

It separates the oscillations of different character: the oscillations of small amplitudes around the equilibrium positions $\pm u_{0}$ are separated by separatrix from the oscillations with the large amplitude around the point $u=0$. Introducing the new variable

$$
z=\tanh \frac{u}{2}
$$

we transform the equations of motion (6) and (7) into

$$
\begin{aligned}
& \left.\frac{\mathrm{d}^{2} z}{\mathrm{~d} \tau^{2}}=\left[\varepsilon^{2}-(1+A)^{2}\right)\right] z^{3}-\left(\varepsilon^{2}-1+A^{2}\right) z, \\
& \left(2 \frac{\mathrm{d} z}{\mathrm{~d} \tau}\right)^{2}=\left[\varepsilon^{2}-(1+A)^{2}\right] z^{4}-2\left(\varepsilon^{2}-1+A^{2}\right) z^{2}+\varepsilon^{2}-(1-A)^{2},
\end{aligned}
$$

respectively. Note that an equation of the same type appears when the sine-Gordon equation is solved, whereas for the Korteweg-de Vries equation only terms up to the third order in $z$ appear [9].

The last equation can be integrated using the Jacobi elliptic functions [6]. To do this we express Eq. (9), in the canonical form of differential equations for the Jacobi elliptic functions [10]:

$$
\begin{aligned}
& \operatorname{sn}(\tau, k) \equiv \operatorname{sn}(\tau), \\
& \operatorname{cn}(\tau, k) \equiv \operatorname{cn}(\tau), \\
& \operatorname{dn}(\tau, k) \equiv \operatorname{dn}(\tau),
\end{aligned}
$$


i.e.

$$
\begin{aligned}
& \left(\frac{\mathrm{d}}{\mathrm{d} \tau} \operatorname{sn}(\tau)\right)^{2}=\left(1-\operatorname{sn}^{2}(\tau)\right)\left(1-k^{2} \operatorname{sn}^{2}(\tau)\right), \\
& \left(\frac{\mathrm{d}}{\mathrm{d} \tau} \operatorname{cn}(\tau)\right)^{2}=\left(1-\mathrm{cn}^{2}(\tau)\right)\left(k_{1}^{2}+k^{2} \mathrm{cn}^{2}(\tau)\right), \\
& \left(\frac{\mathrm{d}}{\mathrm{d} \tau} \operatorname{dn}(\tau)\right)^{2}=\left(1-\mathrm{dn}^{2}(\tau)\right)\left(\mathrm{dn}^{2}(\tau)-k_{1}^{2}\right),
\end{aligned}
$$

where $k$ is the modulus, $0 \leq k \leq 1$, and $k_{1}$ the additional modulus, $k_{1}^{2}=1-k^{2}$ of an elliptic function. Let us remember that the real period of the functions $\operatorname{sn}(\tau, k)$ and $\operatorname{cn}(\tau, k)$ is $4 K(k)$ and for the function $\operatorname{dn}(\tau, k)$ it is $2 K(k)$, where

$$
K \equiv K(k)=\int_{0}^{\pi / 2} \frac{1}{\sqrt{1-k^{2} \sin ^{2} \varphi}} \mathrm{d} \varphi
$$

is the complete elliptic integral of the first kind.

The character of the motion of a particle depends on its energy $E$ (or on the value of the dimensionless energy parameter $\varepsilon(5)$ ). The following particular cases have to be distinguished:

- $0<\varepsilon \leq 1-A$

The particle moves in one of the potential wells (for definiteness in the right). In this case Eq. (9) has the solution

$$
z(\vartheta)=\sqrt{\frac{\varepsilon+1-A}{\varepsilon+1+A}} \operatorname{dn}(\vartheta, k), \quad \vartheta=\frac{1}{2} \sqrt{1-(A-\varepsilon)^{2}} \tau, \quad k^{2}=\frac{4 A \varepsilon}{1-(A-\varepsilon)^{2}} .
$$

Let us remark that due to the relation

$$
\operatorname{dn}(K(k), k)=k^{\prime}=\sqrt{1-k^{2}}=\sqrt{\frac{(\varepsilon+1+A)(1-A-\varepsilon)}{(\varepsilon+1-A)(1+A-\varepsilon)}},
$$

the oscillations of the particle are restricted to the region

$$
\sqrt{\frac{1-A-\varepsilon}{1+A-\varepsilon}} \leq z \leq \sqrt{\frac{\varepsilon+1-A}{\varepsilon+1+A}}
$$

i.e. the particle moves between the turning points of the potential for a given value of the parameter $\varepsilon$.

When the energy of the particle is equal to the height of the potential barrier $\varepsilon=1-A$ (in the phase space the motion is on the separatrix), $k=1$ and

$$
\operatorname{dn}(\vartheta, k=1)=\frac{1}{\cosh \vartheta}
$$

In this case the motion is aperiodic and

$$
\begin{aligned}
& z(\vartheta)=\sqrt{1-A} \frac{1}{\cosh \vartheta}, \\
& \vartheta=\sqrt{A(1-A)} \tau .
\end{aligned}
$$


- $1-A<\varepsilon \leq 1+A$

The energy of the particle is greater than the height of the potential barrier. In this case Eq. (9) has the general solution

$$
\begin{aligned}
& z(\vartheta)=\sqrt{\frac{\varepsilon+1-A}{\varepsilon+1+A}} \operatorname{cn}(\vartheta, k), \\
& \vartheta=\sqrt{A \varepsilon} \tau \\
& k^{2}=\frac{1-(A-\varepsilon)^{2}}{4 A \varepsilon} .
\end{aligned}
$$

In particular limits, the solution (11) behaves as follows:

1. $\varepsilon=1-A, k=1$

$$
\operatorname{cn}(\vartheta, k=1)=\frac{1}{\cosh \vartheta} \text {. }
$$

Hence,

$$
z(\vartheta)=\sqrt{1-A} \frac{1}{\cosh \vartheta}
$$

which coincides with (10).

2. $\varepsilon=1+A, k=0$

$$
\operatorname{cn}(\vartheta, k=0)=\cos \vartheta \text {. }
$$

$$
\begin{aligned}
& z(\vartheta)=\frac{1}{\sqrt{1+A}} \cos \vartheta, \\
& \vartheta=\sqrt{A(1+A)} \tau,
\end{aligned}
$$

describing the periodic motion.

- $\varepsilon>1+A$

In this case Eq. (9) has the solution

$$
\begin{aligned}
& z(\vartheta)=\sqrt{\frac{\varepsilon+1-A}{\varepsilon+1+A}} \operatorname{sn}(\vartheta, k), \\
& \vartheta=\frac{1}{2} \sqrt{(\varepsilon+A)^{2}-1} \tau, \\
& k^{2}=\frac{(\varepsilon-A)^{2}-1}{(\varepsilon+A)^{2}-1} .
\end{aligned}
$$

We notice that for $\varepsilon \rightarrow 1+A, k \rightarrow 0$ and $\operatorname{sn}(\vartheta, k=0)=\sin \vartheta=\cos (\vartheta-\pi / 2)$. In this limit (13) does not coincide with (12), but is shifted by $\pi / 2$. 


\section{Flat bottom potential}

In the special case when $A=1$ the potential (4) is very flat at the bottom and takes the form

$$
V(x)=2 V_{0} \sinh ^{4}\left(\frac{a x}{2}\right) \text {. }
$$

Equations (6) and (7) take the form

$$
\begin{aligned}
& \frac{\mathrm{d}^{2} u}{\mathrm{~d} \tau^{2}}=-4 \sinh ^{3}\left(\frac{u}{2}\right) \cosh \left(\frac{u}{2}\right), \\
& \left(\frac{\mathrm{d} u}{\mathrm{~d} \tau}\right)^{2}=\varepsilon^{2}-4 \sinh ^{4}\left(\frac{u}{2}\right) .
\end{aligned}
$$

The phase portrait is presented in Fig. $2 b$. In this case only the anharmonic oscillations can take place. Equation (9) reads

$$
\left(2 \frac{\mathrm{d} z}{\mathrm{~d} \tau}\right)^{2}=\varepsilon^{2}\left(1-z^{2}\right)-4 z^{2} .
$$

The solutions of this equation in two special cases are the following:

- $0<\varepsilon \leq 2$

$$
\begin{aligned}
& z(\vartheta)=\sqrt{\frac{\varepsilon}{\varepsilon+2}} \operatorname{cn}(\vartheta, k), \quad \vartheta=\sqrt{\varepsilon} \tau, \quad k^{2}=\frac{1}{4}(2-\varepsilon) . \\
& \bullet \varepsilon>2 \\
& z(\vartheta)=\sqrt{\frac{\varepsilon}{\varepsilon+2}} \operatorname{sn}(\vartheta, k), \quad \vartheta=\frac{1}{2} \sqrt{\varepsilon(\varepsilon+2)} \tau, \quad k^{2}=\frac{\varepsilon-2}{\varepsilon+2} .
\end{aligned}
$$

Let us remark that for $\varepsilon=2$ we have $k=0$. Therefore, due to the relations

$$
\begin{aligned}
& \operatorname{cn}(\vartheta, k=0)=\cos \vartheta, \\
& \operatorname{sn}(\vartheta, k=0)=\sin \vartheta
\end{aligned}
$$

we obtain the periodic solutions

$$
z(\vartheta)=\frac{1}{\sqrt{2}} \cos \vartheta \text { or } z(\vartheta)=\frac{1}{\sqrt{2}} \sin \vartheta, \text { where } \vartheta=\sqrt{2} \tau .
$$

The solutions in both considered ranges of variation of the parameter $\varepsilon$ differ only by phase shift.

\section{One-well anharmonic potential}

Finally, we consider the case $A>1$, i.e. single well anharmonic potential. The phase portrait for this potential is presented in Fig. 2c. In this case $\varepsilon>A-1$ and the solution of Eq. (9) has to be considered in two ranges of the parameter $\varepsilon$ :

- $A-1<\varepsilon \leq A+1$

$$
\begin{aligned}
& z(\vartheta)=\sqrt{\frac{\varepsilon+1-A}{\varepsilon+1+A}} \operatorname{cn}(\vartheta, k), \quad \vartheta=\sqrt{A \varepsilon} \tau, \quad k^{2}=\frac{1-(A-\varepsilon)^{2}}{4 A \varepsilon} . \\
& \bullet \varepsilon>A+1 \\
& z(\vartheta, k)=\sqrt{\frac{\varepsilon+1-A}{\varepsilon+1+A}} \operatorname{sn}(\vartheta, k), \quad \vartheta=\frac{1}{2} \sqrt{(\varepsilon+A)^{2}-1} \tau, \quad k^{2}=\frac{(\varepsilon-A)^{2}-1}{(\varepsilon+A)^{2}-1} .
\end{aligned}
$$

In this case the solutions seem to have the same character as in the preceding cases, but in fact it turns out not to be the case (see Sec. 6). 


\section{Reversed potential}

It is interesting to consider the case when $V_{0} \rightarrow-V_{0}$, i.e. the potential $V(x)=-\frac{1}{2} V_{0}(A \cosh a x-1)^{2}$,

where $V_{0}>0$ and $0<A<1$ (see Fig. 3a).
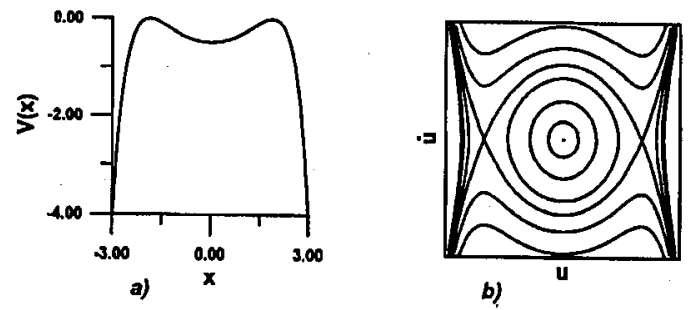

Fig. 3. The potential (14) (a) and the phase portrait for this potential (b).

We investigate the case when the energy of the particle is nonpositive, $E \leq 0$, leaving the notation unchanged with one exception

$$
\varepsilon=\sqrt{\frac{2|E|}{V_{0}}} .
$$

The equation of motion and energy equation have the form

$$
\begin{aligned}
& \frac{\mathrm{d}^{2} u}{\mathrm{~d} \tau^{2}}=A \sinh u(A \cosh u-1), \\
& \left(\frac{\mathrm{d} u}{\mathrm{~d} \tau}\right)^{2}=(A \cosh u-1)^{2}-\varepsilon^{2} .
\end{aligned}
$$

The phase portrait of the system is presented in Fig. $3 \mathrm{~b}$. We see that the system has the following critical points:

- the centre: $u=0, \dot{u}=0$,

- two saddle points: $u= \pm u_{0}, \dot{u}=0$, where $\cosh u_{0}=\frac{1}{A}$.

We consider only the case

$$
-\frac{1}{2} V_{0}(1-A)^{2}<E \leq 0, \quad \text { i.e. } \quad 0 \leq \varepsilon<1-A,
$$

when the oscillations of the particle within the well take place.

When $\varepsilon=0$ the particle moves on the separatrix

$\dot{u}^{2}=(A \cosh u-1)^{2}$.

The points, where the separatrix intersect the $u$ axis, correspond to the points of local maximum of the potential.

Using the variable

$$
z=\tanh \frac{u}{2}
$$


we rewrite Eq. (16) in the form

$$
\left(\frac{\mathrm{d} z}{\mathrm{~d} \tau}\right)^{2}=(1+A-\varepsilon)(1-A+\varepsilon)\left(z^{2}-z_{1}^{2}\right)\left(z^{2}-z_{2}^{2}\right)
$$

where

$$
\begin{aligned}
& z_{1}^{2}=\frac{1-(A-\varepsilon)^{2}}{(1+A)^{2}-\varepsilon^{2}}=\frac{1-A+\varepsilon}{1+A+\varepsilon} \\
& z_{2}^{2}=\frac{1-(A-\varepsilon)^{2}}{(1+A)^{2}-\varepsilon^{2}}=\frac{1-A-\varepsilon}{1+A-\varepsilon} .
\end{aligned}
$$

As $0<\varepsilon<1-A, \quad 1>z_{1}^{2}>z_{2}^{2}>0$

and Eq. (17) can be rewritten in the form

$$
\left(\frac{\mathrm{d}}{\mathrm{d} \vartheta} \frac{z}{z_{2}}\right)^{2}=\left[1-\left(\frac{z}{z_{2}}\right)^{2}\right]\left[1-k^{2}\left(\frac{z}{z_{2}}\right)^{2}\right],
$$

where we have introduced the new variable

$$
\vartheta=\frac{1}{2} \sqrt{1-(A-\varepsilon)^{2}} \tau
$$

and

$$
k^{2}=\frac{z_{2}^{2}}{z_{1}^{2}}=\frac{1-(A+\varepsilon)^{2}}{1-(A-\varepsilon)^{2}} \leq 1
$$

It is clear that this equation is of the type

$$
\left(\frac{\mathrm{d} f}{\mathrm{~d} \vartheta}\right)^{2}=\left(1-f^{2}\right)\left(1-k^{2} f^{2}\right)
$$

and its solution is

$$
z(\vartheta)=z_{2} \operatorname{sn}(\vartheta, k)
$$

or in explicit form

$$
z(t)=\sqrt{\frac{1-A-\varepsilon}{1+A-\varepsilon}} \operatorname{sn}\left(\frac{1}{2} \omega_{0} \sqrt{1-(A-\varepsilon)^{2}} t, k\right) .
$$

Let us consider the limit when $\varepsilon=0$, i.e. the motion of the particle on the separatrix. In this case $k^{2}=1$ and

$$
\operatorname{sn}(\vartheta, k=1)=\tanh \vartheta
$$

Therefore the solution takes the form

$$
z(t)=\sqrt{\frac{1-A}{1+A}} \tanh \left(\frac{1}{2} \omega_{0} \sqrt{1-A^{2} t}\right) .
$$

In the other limit $\varepsilon=1-A$, we have the trivial solution $z(t)=0$, i.e. the particle is at rest in the equilibrium position at the bottom of the potential well. 


\section{Discussion}

Solutions of the equations of motion (2) for the potentials (4) and (14) are obtained by inverting the formula (8) using (5), which gives

$$
x(t)=\frac{2}{a} \operatorname{atanh} z(t) .
$$

The results are given for these potentials in Table I and Table II, respectively. Let us shortly discuss their properties.

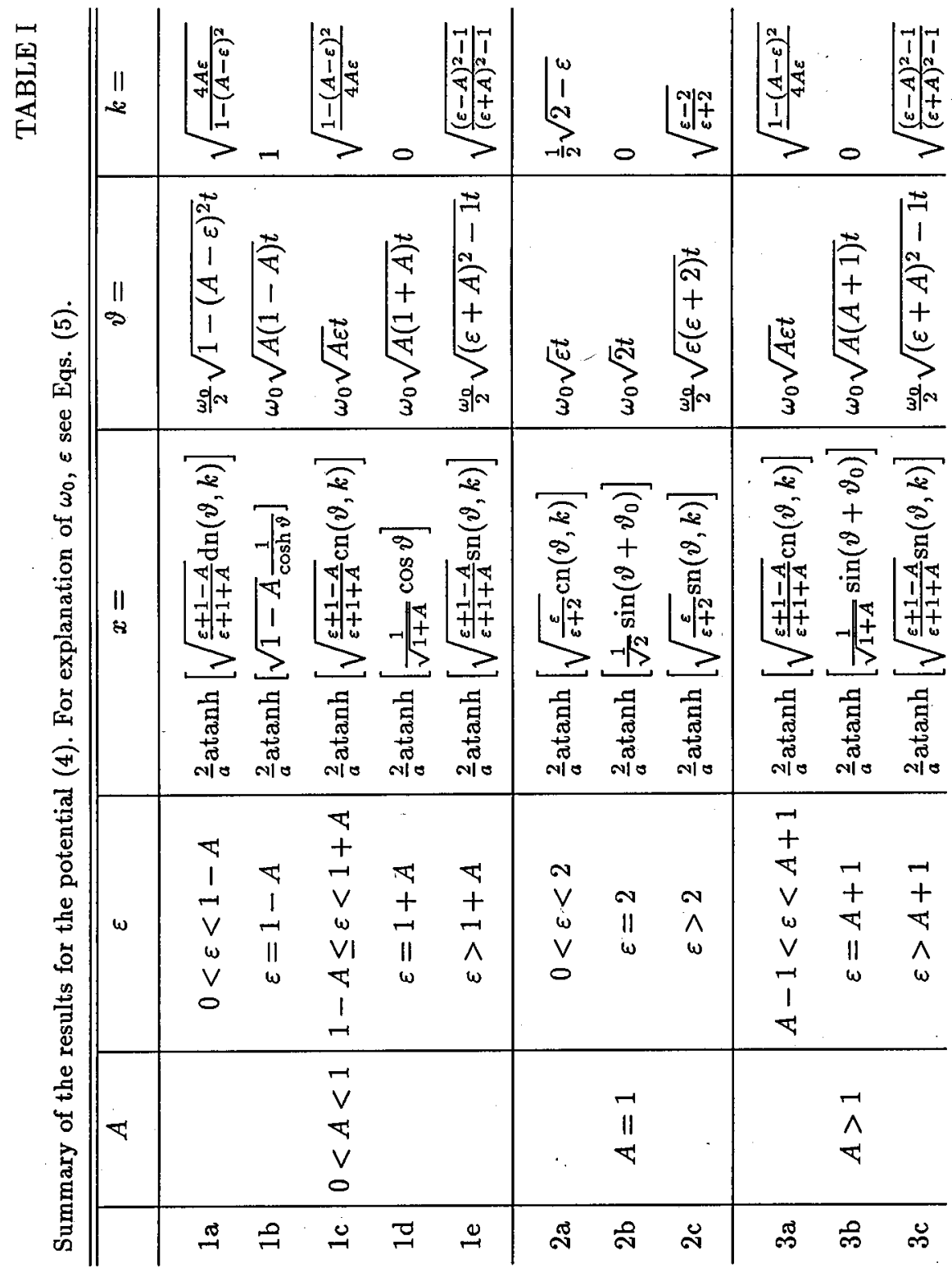


TABLE II

Summary of the results for the reversed potential (14). For explanation of $\omega_{0}, \varepsilon$ see Eqs. (5) and (15). For all case $1 \mathrm{a}-\mathrm{c} 0<A<1$.

\begin{tabular}{c|c|l|c|c}
\hline \hline & \multicolumn{1}{|c|}{$\varepsilon$} & \multicolumn{1}{|c|}{$x=$} & $\vartheta=$ & $k=$ \\
\hline $1 \mathrm{a}$ & $0<\varepsilon<1-A$ & $\frac{2}{a} \operatorname{atanh}\left[\sqrt{\frac{1-A-\varepsilon}{1+A-\varepsilon}} \operatorname{sn}(\vartheta, k)\right]$ & $\frac{\omega_{0}}{2} \sqrt{1-(A-\varepsilon)^{2}} t$ & $\sqrt{\frac{1-(A+\varepsilon)^{2}}{1-(A-\varepsilon)^{2}}}$ \\
$1 \mathrm{~b}$ & $\varepsilon=0$ & $\frac{2}{a} \operatorname{atanh}\left[\sqrt{\frac{1-A}{1+A}} \tanh \vartheta\right]$ & $\frac{\omega_{0}}{2} \sqrt{1-A^{2} t}$ & 1 \\
$1 \mathrm{c}$ & $\varepsilon=1-A$ & $x(t)=0$ & - & -
\end{tabular}

In the case of double-well potential, $0<A<1$, the motion of a particle of energy lower than the height of the potential hump, $\varepsilon^{2}<(1-A)^{2}$, is qualitatively close to harmonic oscillations, which is still true even for relatively high energies, as it is shown in Fig. 4a.
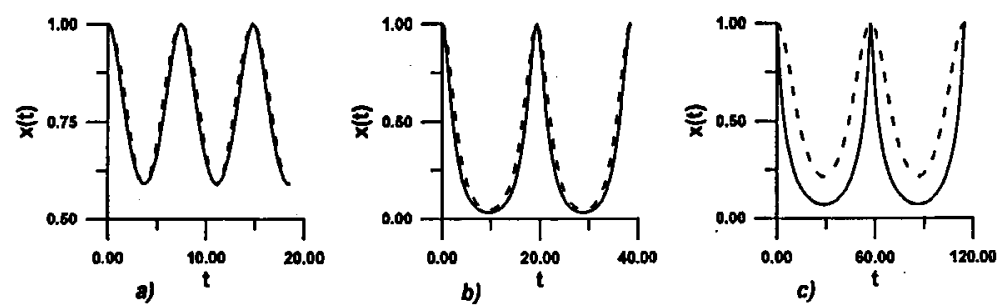

Fig. 4. The solutions of the equations of motion for $\varepsilon<1-A$ (the right well is chosen); (a) $A=0.3, \varepsilon=0.4$ (solid line), compared to the harmonic oscillation of the same period and amplitude (dashed line); (b) $A=0.3, \varepsilon=0.699$ (solid line), compared to the analogous oscillations in the potential of the type (1) of the same modulus $k$ (dashed line); (c) $A=0.01, \varepsilon=0.989$ (solid line), compared to the analogous oscillations in the potential (1) of the same modulus $k$ (dashed line).

The character of motion changes only as $\varepsilon$ approaches $1-A$, exhibiting the characteristic features of Jacobi delta amplitude function (see Fig. 4b). The solutions of this type are characteristic for double-well potentials; as an example, we compare the solution for the potential (4) with the solutions for the potential (1) (cf. e.g. [1])

$$
x(t)=C \operatorname{dn}(\omega t, k) .
$$

The difference between the potential (4) and the potential (1) becomes important when the amplitude of motion in terms of $z$ approaches 1 , because of the properties of the atanh function. The solution of the equations of motion for $A=0.01$ is shown in Fig. 4c.

Figure 5 shows the aperiodic solution of the problem for $\varepsilon=1-A$.

When the energy exceeds the height of the inner barrier, $1-A \leq \varepsilon \leq 1+A$, the character of the solutions obviously changes. The solutions for energies slightly 


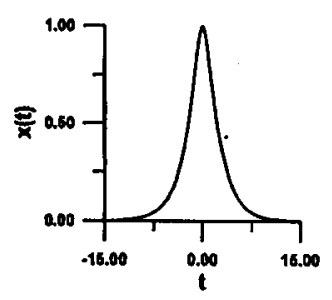

Fig. 5. The movement of a particle in the case $\varepsilon=1-A$.
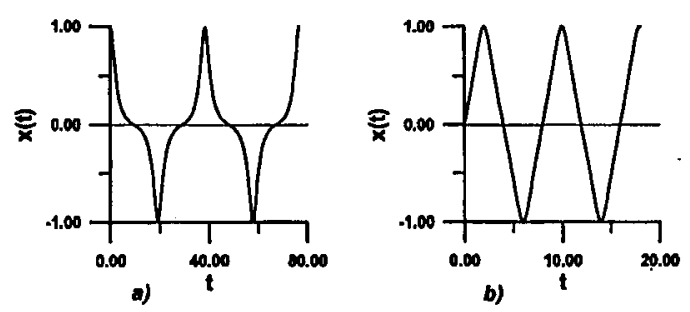

Fig. 6. The movement of a particle with the energy $\varepsilon>1-A$ in the potential defined by $A=0.3$; (a) $\varepsilon=0.701$; (b) $\varepsilon=1.7$.

higher than the potential hump expressed in terms of the $z$ variable have the form of Jacobi cosine amplitude function with modulus close to 1 . The characteristic form of this solution is shown in Fig. 6a. For higher energies, the solutions have the form of Jacobi sine amplitude function with the modulus $k$ approaching 1 , as the energy increases to infinity. This constitutes a considerable difference in comparison with the $\phi^{4}$ problem. Note that

$$
\operatorname{sn}(x, k) \rightarrow \tanh (x) \text { for } k \rightarrow 1 \text {. }
$$

According to the corresponding formulae (Table I, n.1e), the function $x(t)$ in this limit becomes nearly linear, whereas the solution of the $\phi^{4}$ problem has the form of Jacobi cosine amplitude function with $k \rightarrow 1 / \sqrt{2}$ as $\varepsilon \rightarrow \infty$. The solution in this case is shown in Fig. 6b. It is a characteristic feature of the potential (4) that in the limit of infinite energies the oscillations resemble the motion of a particle in an infinite square well.

The solutions for the flat bottom potential (Table I n.2) for low energies have the form of atanh of Jacobi cosine amplitude function with the modulus $k<1 / \sqrt{2}$.

For the strongly anharmonic single-well potential, the solutions in lower range of energies have the form of Jacobi cosine amplitude function, slightly influenced by the atanh function, with the modulus $k$ close to zero for low energies, then growing to certain maximal value and decreasing again to zero for $\varepsilon=A+1$ (Table I n.3).

The solutions for high energies are the same for all the potential types.

Figure 7 a shows the solution for the reversed potential (14), (see Table II), when the energy is well below 0. On the next figure (Fig. 7b) the solution for $\varepsilon$ close to 0 is shown. The solution exhibits the typical shape of Jacobi sine amplitude function. The influence of the atanh transformation is rather small for this value 

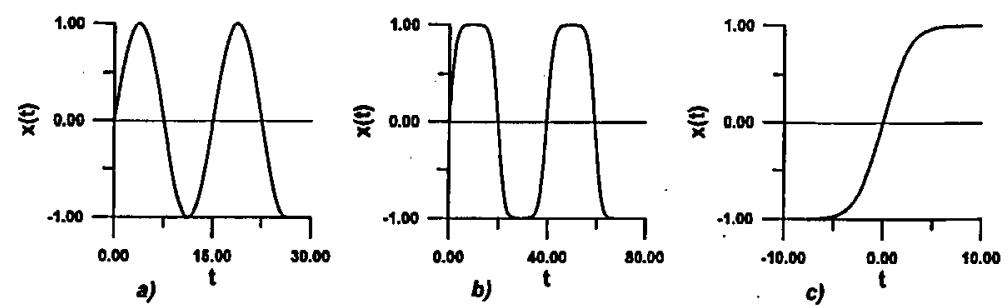

Fig. 7. The movement of a particle in the reversed potential, $A=0.3$; (a) Energy considerably lower than $0, \varepsilon=0.4$; (b) Energy close to $0, \varepsilon=0.01$; (c) The aperiodic movement with $\varepsilon=0$.

of $A$. Figure 7c contains the solutions for $\varepsilon=0$. In this case, the system shows aperiodic behaviour.

\section{Conclusions}

In this paper the strongly anharmonic one-particle potential (4) has been studied in the classical limit. The exact solution of the equation of motion was found in the whole range of model parameter covering the cases of double-well and single-well potential. Despite the general similarity to the well-known $\phi^{4}$ potential it seems worthwhile to underline some characteristic differences between these two potentials which might be important from the point of view of their applications.

The potential barrier in the double-well case, $A<1$, becomes wide and flat in the limit of $A \rightarrow 0$ (see (4)). Although this does not influence the classical solution it would be essential in the quantum limit for exactness of the quasiclassical approximation. Similarly, for increasing energies the exponential character of the potential (4) becomes apparent, leading to nearly free motion between the turning points. This suggests that in the quantum limit the spectrum should resemble that of an infinite square well.

- Finally, let us remark that the results of the present paper can be applied in several solitonic problems, like classical theory of the hydrogen bond (see e.g. [11]) and in the theory of hydrogen bonded linear chains [12]. It is also interesting to compare the result (10a) with solutions appearing in the theory of $\mathrm{KdV}$ and modified $\mathrm{KdV}$ equation, where the functions (cosh $a \zeta)^{-2}$ (corresponding to the Pöshl-Teller potential) and (cosh $a \zeta)^{-1}$ appear [13-15]. Both of these functions correspond to reflectionless potentials. This may allow the inverse scattering method to approach to the soliton dynamics of chains with the potential (4). This will be examined in future works.

\section{References}

[1] A. Radosz, J: Phys. C, Solid State Phys. 18, L189 (1985). .

[2] H. Konwent, Phys. Lett. A 118, 467 (1986).

[3] E. Matsushita, T. Matsubara, Progr. Theor. Phys. (Kyoto) 67, 1 (1982); M.C. Lawrence, G.N. Robertson, Ferroelectrics 34, 179 (1981); G.N. Robertson, M.C. Lawrence, J. Phys. C, Solide State Phys. 14, 4599 (1981). 
[4] M. Razavy, Am. J. Phys. 48, 285 (1980).

[5] O.B. Zaslavsky, V.V. Ulyanov, Zhurn. Eksp. Teor. Phys. 87, 1724 (1984).

[6] O.B. Zaslavsky, V.V. Ulyanov, Teor. Mat. Fiz. 71, 260 (1987).

[7] A.V. Turbiner, Zhurn. Exp. Teor. Phys. 94, 33 (1988).

[8] H. Konwent, P. Machnikowski, A. Radosz, J. Phys. A 28, 3757 (1995).

[9] G. Eilenberger, Solitons, Mathematical Methods for Physicists, Springer, Berlin 1981.

[10] P.F. Byrd, M.D. Friedman, Handbook of Elliptic Integrals for Engineers and Physicists, Springer, Berlin 1954.

[11] Wen Ge Han, Chun-Ting Zhang, J. Phys., Condens. Matter 3, 27 (1991).

[12] H. Konwent, P. Machnikowski, A. Radosz; submitted to J. Phys., Condens. Matter.

[13] M. Wadati, J. Phys. Soc. Jpn. 33, 673 (1975).

[14] R. Jackiw, Rev. Mod. Phys. 49, 681 (1977).

[15] R.K. Dodd, J.C. Eilbeck, J.D. Gibbon, H.C. Harris, Solitons and Nonlinear Wave Equations, Academic Press, New York 1982, Chs. 2, 3. 